\title{
The proposal of Paediatric Virology and its perspectives: An interview with Professor of Paediatrics Maria Theodoridou
}

\author{
IOANNIS N. MAMMAS and DEMETRIOS A. SPANDIDOS
}

Department of Clinical Virology, School of Medicine, University of Crete, Heraklion 71003, Greece

Received July 18, 2017; Accepted August 22, 2017

DOI: $10.3892 /$ etm.2017.5009

\begin{abstract}
Professor Maria Theodoridou, Emeritus Professor of Paediatrics at the University of Athens, is one of the few paediatricians in Greece, who have experienced almost all the infectious diseases of the second half of the 20th century and their severe consequences, prior to the widespread adoption of immunisations. A milestone during her career was the establishment of a specialised National Reference Unit for the care of paediatric patients with acquired immune deficiency syndrome (AIDS) at the 'Aghia Sophia' Children's Hospital in Athens, Greece. According to Professor Theodoridou, training on the prevention, management and treatment of neonatal and paediatric viral infections represents a new educational challenge for both community as well as hospital-based paediatric health professionals. The debate of the potential strategically principal role of Paediatric Virology subspecialists in the primary, secondary and tertiary clinical practice is definitely necessary and needs further discussion and evaluation, she adds. She describes the difficulties that Greece, a country under a long-standing financial crisis, faces for the hospital-based management of paediatric viral infections and refers to the future advances, which are expected in the field of diagnosis and treatment of viral infections in neonates and children. In the context of the 3rd Workshop on Paediatric Virology, which will be held in Athens on October 7th, 2017, Professor Theodoridou will focus on the immigration crisis and vaccination policy.
\end{abstract}

\section{Contents}

1. Introduction

2. Questions and Answers

Correspondence to: Professor Demetrios A. Spandidos, Department of Clinical Virology, School of Medicine, University of Crete, Heraklion 71003, Greece

E-mail: spandidos@spandidos.gr

Key words: Paediatric Virology, poliomyelitis, HIV, immigration, Maria Theodoridou

\section{Introduction}

Professor Maria Theodoridou (Fig. 1), Emeritus Professor of Paediatrics at the University of Athens School of Medicine, was born in Alexandria, Egypt; she completed her primary and secondary education in Athens, Greece (1). She studied Medicine at the University of Athens School of Medicine, where she entered in 1963. A crucial turning point in her career was her experience as a senior medical student at the 1st Department of Paediatrics of the University of Athens at the 'Aghia Sophia' Children's Hospital under the direction of Professor Nikolaos Matsaniotis, where she admired the mode of paediatric thinking, working and contribution. During her term as a paediatric resident, she came to realise the importance of paediatric infections as well as the value of their prevention through immunisations. In 1974, she applied and obtained a scholarship for studies on Virology from the National Scholarship Foundation in order to attend the Institute of Child Health at the University of London (London, UK) and work on congenital infections (1974-1976). Following her return to Greece, she had the opportunity to serve for more than 35 years at the Paediatric Infectious Diseases Unit (MAKKA) at the 'Aghia Sophia' Children's Hospital, where in 1997 she became Chief of the Unit (1997-2012). Professor Theodoridou is vice President of the National Immunisation Committee of Greece, former President of the Hellenic Paediatric Society of Infectious Diseases, President of the National Committee for Polio Eradication and President of the National Committee for Measles and Rubella Elimination at the Hellenic Centre for Disease Control and Prevention (HCDCP) in Greece.

Professor Theodoridou remains one of the few paediatricians in Greece, who have experienced almost all the infectious diseases of the second half of the 20th century and their severe consequences, prior to the widespread adoption of immunisations. A milestone during her career was the evolution of acquired immune deficiency syndrome (AIDS) and the establishment of a specialised National Reference Unit for the care of paediatric patients infected with human immunodeficiency virus (HIV) at the 'Aghia Sophia' Children's Hospital in Athens, Greece. Her main academic and research fields of interest are paediatric viral infectious diseases, congenital infections, vaccinations and paediatric AIDS. She is one of the principal academics at the Paediatric Virology Study Group (PVSG), since its birth (2). She has chaired the 1st and the 2nd Workshop on Paediatric Virology held in Athens in 2015 and 2016, where 
she has given her key lectures on poliomyelitis 60th anniversary and children's HIV experience in Greece, respectively (1,3-5). She has also actively worked on the proposal of Paediatric Virology as a paediatric subspecialty, which was published in 2016 (6). To date, she has been awarded by the PVSG with the '2015 Paediatric Virology Award in Paediatrics' and the '2016 Paediatric Virology Award in HIV' for her outstanding academic and clinical research and her exceptional teaching and publishing contribution in the management of paediatric infectious diseases in Greece and the creation of the first specialised Paediatric HIV Unit in Athens, Greece $(1,4)$. In the context of the 3rd Workshop on Paediatric Virology, which will be held in Athens on October 7th, 2017 (7), Professor Maria Theodoridou will focus on the immigration crisis and vaccination policy.

\section{Questions and Answers}

Question: Professor Maria Theodoridou, first of all, thank you for your support all these years. Since our proposal has gained the interest of several worldwide experts on the scientific fields of Neonatology, Paediatrics, Paediatric Infectious Diseases and Virology, we would like to have your comment on the potential role of Paediatric Virology as a new paediatric subspecialty.

Answer: Training on the prevention, management and treatment of neonatal and paediatric viral infections represents a new educational challenge for both community as well as hospital-based paediatric health professionals. Due to the current advances on the recently defined scientific field of Paediatric Virology, this requires a state-of-the-art continuous medical education on viral infections provided by both clinicians and basic scientists. The debate of the potential strategically principal role of Paediatric Virology subspecialtists in the primary, secondary and tertiary clinical practice is definitely necessary and needs further discussion and evaluation. In the future, it is important to determine whether and how Paediatric Virology as a new paediatric subspecialty can indeed help paediatric clinical practice allowing the development of accredited training programmes by tertiary paediatric infectious diseases centres able to attract the highest quality paediatric trainees.

Question: Your plenary lecture during the 1st Workshop on Paediatric Virology in Athens, Greece was focused on polio, a re-emerging life-treating disease. I would like to have your statement on the occasion of the 60th-year vaccine anniversary against poliomyelitis, which was celebrated in 2015.

Answer: Poliomyelitis is an acute infectious disease of childhood, which is caused by small RNA viruses of the enterovirus group of the Picornaviridae family. To date, three antigenically distinct strains are known, 1, 2 and 3, with type 1 accounting for around $85 \%$ of cases. The clinical manifestations of the disease vary greatly; in most cases they are mild, while paralytic illness is rare, affecting less than $1 \%$ of the infected children. The year 2015 was the 60th anniversary of the Jonas Salk's inactivated polio vaccine (IPV), which enabled children to be protected against poliomyelitis, the crippling disease. With the development of the oral polio vaccine (OPV) by Albert Sabin in 1961, the world was given the tools to both stop outbreaks and strengthen and built immunity to ensure

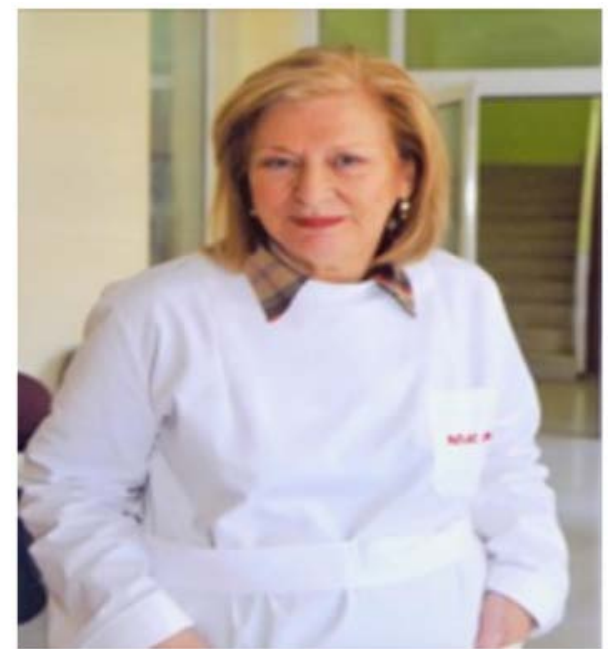

Figure 1. Professor Maria Theodoridou, Emeritus Professor of Paediatrics at the University of Athens (Athens, Greece).

that children could grow up without the threat of polio. The implementation of OPV and IPV into clinical practice led to the eradication of polio in the Americas, in the Western Pacific and Europe. Currently, $80 \%$ of the world's population live in polio-free regions. However, Pakistan, Afghanistan and Nigeria are countries where polio is still categorised as an endemic viral infection. During the period of 2013-2014, an upsurge of polio in areas that had thought to have been free of the disease appeared. The confirmed circulation of the wild-type poliovirus (WPV) in Israel and the outbreak of acute flaccid paralysis (AFP) in Syria mean that there is a high risk the disease to be reintroduced into Europe. All member states of the European Union (EU) should implement a prevention policy, which is based on enhancing the vaccination of resident and refugee populations, strengthening surveillance and being prepared to respond quickly to the identification of polio. In Greece, there is already a national action plan, which includes programmes to sustain high levels of polio immunisation coverage, AFP surveillance and actions in the event of a suspected or confirmed poliomyelitis case. Fighting polio with vaccination has been one of the most successful Public Health programmes in history, reducing the number of polio cases by $99 \%$, thus making the expectation towards disease eradication possible.

Question: One of the most significant milestones during your career was the establishment of the first specialised National Reference Unit in Greece for the care of paediatric patients with AIDS at the 'Aghia Sophia' Children's Hospital in Athens, Greece. How difficult was this attempt?

Answer: The emergence of HIV-1 infection in children in 1982 was a new problem of unknown dimensions in all countries. In Greece, the diagnosis of the first cases was concerning multi-transfused children and adolescents with thalassaemia and haemophilia, who had been infected. The magnitude of the problem of children vertically infected was unknown, although the incidence of infection among women was low, less than 1:10,000. Actually, the number of infected babies was small, and still remains so, with the implementation of preventive strategy with zidovudine and antiretroviral treatment 
during pregnancy and the neonatal period. The 'new infectious disease' enforced the establishment of a specialised Paediatric AIDS Unit in the 'Aghia Sophia' Children's Hospital, which became the reference centre for Greece. The Unit had a multifaceted goal. The main activity was the care of the paediatric patients and the follow-up of their treatment, but also the social and psychological support of the children's parents and relatives. In addition, efforts were taken in collaboration with adults AIDS Units and maternity clinics for the prompt management of the HIV-positive pregnant women and their newborns. All babies had a follow-up of 2 years. Of great importance was also the educational role of our Unit for the healthcare personnel, students and public, mainly during the period of unbased fears and prejudices. In a rather short period of time, due to scientific achievements in all fields, the prognosis of HIV infection has changed. Unfortunately, children and adolescents of the AIDS initial period did not have the benefaction of the antiretroviral therapy. Today, children grow up to adulthood and demand their rights for life and hope.

Question: Over the last years Europe faces an unprecedented immigration crisis, with a significant impact on children's life and health. Greece, one of the principal gateway countries of the EU has experienced an increased inflow of refugees and migrants arriving by sea in the Greek islands. What were the main actions that took place in Greece regarding its vaccination policy towards refugees and migrants children?

Answer: According to the World Health Organisation (WHO), hosting countries should ensure appropriate vaccinations among refugees and migrants irrespective of their legal status. In Greece, an imperative need emerged for implementing supplementary immunisation activities, particularly to refugee children living in the 'Points of care', all over the country. Vaccinations were conducted in the form of a mass campaign by non-governmental organisations (NGOs). The efforts were performed under the coordination of the General Secretariat for Public Health of the Hellenic Ministry of Health. In this framework, the recommendations on refugees and migrants vaccination of the National Immunisation Committee were followed and more than 30,000 vaccines were administered. According to data collected by the Hellenic Ministry of Health, the children were vaccinated for 10 infectious diseases and their estimated immunisation coverage with 1st swoop ranged between 75 and $83 \%$. Taking into account the difficulties even for the vaccines' supply, the above result is really encouraging. Public Health Authorities and NGOs have indeed worked hard together in order to protect from infectious diseases children living through one of the biggest crises of our times. The challenge, today, is to continue immunisation of refugees and migrants in Greece, as part of routine primary health care and develop a plan for providing immunisation to all newly arrived refugees and migrants.

Question: Since 2010, Greece is under a long-standing financial crisis. How this crisis has changed your hospital-based clinical practice on the management of paediatric infectious diseases in Greece?
Answer: Due to the current financial crisis, the number of children, who attend the emergency paediatric departments of the public secondary and tertiary hospitals in Athens, has been significantly increased. Moreover, a high percentage of paediatric patients has shifted from private doctors to public primary health care centres, all over the country. This has resulted in increased hospital admissions. Regarding infectious diseases, due to the financial shortage mainly of nursing staff, our independent infectious diseases Unit at the 'Aghia Sophia' Children's Hospital in Athens, Greece, was temporarily closed and children with infectious diseases are treated in single rooms of the main hospital.

Question: What challenges and advances on paediatric viral infections are expected in the future? How are these advances expected to change our paediatric clinical practice?

Answer: Future advances are definitely expected in the field of diagnosis and treatment of viral infections in neonates and children. Molecular Biology and Genetics will facilitate the prompt and less expensive diagnosis of more viral infections in our little patients. New antiviral drugs and improvement of other existing ones will be available as well as new vaccines for the prevention of 'old' and emerging viral infections. An important challenge is to maintain the successful eradication of the infectious diseases of the past as small pox and polio, adopting an effective strategy for vaccine hesitancy. Another challenge is for the ever present threat of influenza, for which a global preparedness is mandatory. Continuous education is needed for virologists for the newly recognised diseases as well as for future paediatricians for the prudent use of antiviral drugs, in order to avoid the development of their resistance.

Question: Thank you for your time, as well as for your valuable contribution at the 3rd Workshop on Paediatric Virology on October in Athens.

\section{References}

1. Greenough A, Theodoridou M, Kramvis A, Mammas IN, Christaki I, Koutsaftiki C, et al: Workshop on Paediatric Virology. Paediatric Virology Study Group, Athens, October 10, 2015.

2. Mammas IN, Theodoridou M and Spandidos DA: The development of the Paediatric Virology Study Group: Ten years in the making. Exp Ther Med 13: 363, 2017.

3. Mammas IN, Greenough A, Theodoridou M, Kramvis A, Christaki I, Koutsaftiki C, Koutsaki M, Portaliou DM, Kostagianni G, Panagopoulou P, et al: Current views and advances on Paediatric Virology: An update for paediatric trainees. Exp Ther Med 11: 6-14, 2016.

4. Thiagarajan P, Gardner S, Theodoridou M, Kramvis A, Melidou A, Papaioannou G, et al: 2nd Workshop on Paediatric Virology. Paediatric Virology Study Group, Athens, October 8, 2016.

5. Mammas IN, Theodoridou M, Kramvis A, Thiagarajan P, Gardner S, Papaioannou G, Melidou A, Koutsaki M, KostagianniG, Achtsidis V, et al: Paediatric Virology: A rapidly increasing educational challenge. Exp Ther Med 13: 364-377, 2017.

6. Mammas IN, Greenough A, Theodoridou M and Spandidos DA: Paediatric Virology: A new paediatric subspecialty? A proposal at the Workshop on Paediatric Virology, Athens, October 10, 2015. Exp Ther Med 11: 3-5, 2016.

7. Mammas IN and Spandidos DA: Athens-based meeting to discuss the paediatric virology crossroad in October 2017. Acta Paediatr 106: 1536, 2017. 